\title{
Bone lesions in Chinese POEMS syndrome patients: imaging characteristics and clinical implications
}

\author{
Fengdan Wang ${ }^{1}$, Xufei Huang ${ }^{2}$, Yan Zhang ${ }^{1}$, Jian Li $^{3}{ }^{3}$ Daobin Zhou ${ }^{3}$, Zhengyu Jin ${ }^{\text {Corresp. }}{ }^{1}$ \\ 1 Department of Radiology, Peking Union Medical College Hospital, Beijing, China \\ 2 Class of 2009, Peking Union Medical College, Beijing, China \\ 3 Department of Hematology, Peking Union Medical College Hospital, Beijing, China \\ Corresponding Author: Zhengyu Jin \\ Email address: pumchradiology@126.com
}

Objective. Bone lesion is crucial for diagnosing and management of polyneuropathy, organomegaly, endocrinopathy, monoclonal protein, and skin change (POEMS) syndrome, a rare plasma cell disorder. This study is to compare the effectiveness of X-ray skeletal survey (SS) and computed tomography (CT) for detecting bone lesions in Chinese POEMS syndrome patients, and to investigate the relationship between bone lesion features and serum markers. Methods. SS and chest/abdomen/pelvic CTimages of 38 Chinese patients (26 males, 12 females, aged 21-70 years) with POEMS syndrome recruited at our medical center between January 2013 and January 2015 were retrospectively analyzed. Bone lesions identified by CT were further categorized according to the size $(<5 \mathrm{~mm}, 5-10 \mathrm{~mm}$, $>10 \mathrm{~mm}$ ) and appearance (osteosclerotic, lytic, mixed). The percentage of plasma cells in bone marrow smears, type of immunoglobulin, platelet (PIt), and levels of serum bone metabolic markers and inflammatory factors including alkaline phosphatase (ALP), calcium, phosphate, parathyroid hormone (PTH), beta-isomerized C-telopeptide ( $\beta$-CTx), vascular endothelial growth factor (VEGF), and interleukin (IL)-6 levels were also recorded. Results. Of the 38 POEMS syndrome patients, the immunoglobulin heavy chain isotypes were IgA in 25 patients $(65.8 \% ; 25 / 38)$ and IgG in 13 patients $(34.2 \% ; 13 / 38)$, and the light chain isotypes were $\lambda$ in 35 patients (92.1\%; 35/38) and $\mathrm{k}$ in 3 patients (7.9\%; 3/38). There were 23 patients with thrombocytosis. More patients with bone lesions were detected by CT than by SS ( $97.4 \%$ vs. $86.8 \%)$. The most commonly affected location was the pelvis $(89.5 \%)$, followed by the spine, clavicle/scapula/sternum/ribs, skull, and long bones. Of the 38 POEMS syndrome patients, 35 (94.6\%) had osteosclerotic and 32 (86.5\%) had mixed lesions. Osteosclerotic lesions were typically scattered, variable in size, and plaque-like, whereas mixed lesions were pouch-shaped or soup bubble-like with a clear sclerotic margin and were generally larger. Although the majority of bone lesions were small in size, $23(62.2 \%)$ had at least one lesion $>10 \mathrm{~mm}$. There was no correlation between serum 
marker levels and bone lesion patterns after Bonferroni correction (all $P>0.001$ ).

Conclusions. CT is more sensitive and accurate than SS in detecting bone lesions in POEMS syndrome. 


\section{Bone lesions in Chinese POEMS syndrome patients: imaging}

\section{2 characteristics and clinical implications}

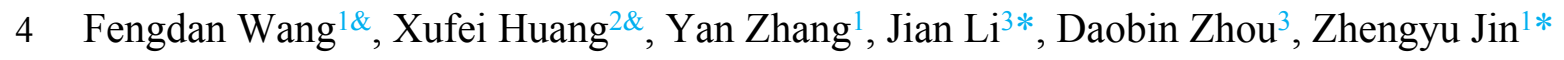
5

$6{ }^{1}$ Department of Radiology, Peking Union Medical College Hospital, Chinese Academy of

7 Medical Sciences \& Peking Union Medical College, Beijing, China

$8{ }^{2}$ Class of 2009, Peking Union Medical College, Beijing, China

$9{ }^{3}$ Department of Hematology, Peking Union Medical College Hospital, Chinese Academy of

10 Medical Sciences \& Peking Union Medical College, Beijing, China

\& These authors contributed equally to this work.

*Corresponding authors

15 Jian Li

16 \#1 Shuaifuyuan, Dongcheng District, Beijing, 100730, China.

17 Phone: +86-10-69155020

18 E-mail: LiJian@pumch.cn

Zhengyu Jin

21 \#1 Shuaifuyuan, Dongcheng District, Beijing, 100730, China.

Phone: $+86-10-69159608$

E-mail: pumchradiology@126.com 


\section{ABSTRACT}

Objective. Bone lesion is crucial for diagnosing and management of polyneuropathy, organomegaly, endocrinopathy, monoclonal protein, and skin change (POEMS) syndrome, a rare plasma cell disorder. This study is to compare the effectiveness of X-ray skeletal survey (SS) and computed tomography (CT) for detecting bone lesions in Chinese POEMS syndrome patients, and to investigate the relationship between bone lesion features and serum markers.

Methods. SS and chest/abdomen/pelvic CT images of 38 Chinese patients (26 males, 12 females, aged 21-70 years) with POEMS syndrome recruited at our medical center between January 2013 and January 2015 were retrospectively analyzed. Bone lesions identified by CT were further categorized according to the size $(<5 \mathrm{~mm}, 5-10 \mathrm{~mm},>10 \mathrm{~mm})$ and appearance (osteosclerotic, lytic, mixed). The percentage of plasma cells in bone marrow smears, type of immunoglobulin, platelet (Plt), and levels of serum bone metabolic markers and inflammatory factors including alkaline phosphatase (ALP), calcium, phosphate, parathyroid hormone (PTH), beta-isomerized Ctelopeptide ( $\beta$-CTx), vascular endothelial growth factor (VEGF), and interleukin (IL)-6 levels were also recorded.

Results. Of the 38 POEMS syndrome patients, the immunoglobulin heavy chain isotypes were IgA in 25 patients $(65.8 \% ; 25 / 38)$ and $\operatorname{IgG}$ in 13 patients $(34.2 \% ; 13 / 38)$, and the light chain isotypes were $\lambda$ in 35 patients $(92.1 \% ; 35 / 38)$ and $\kappa$ in 3 patients $(7.9 \% ; 3 / 38)$. There were 23 patients with thrombocytosis. More patients with bone lesions were detected by CT than by SS (97.4\% vs. $86.8 \%)$. The most commonly affected location was the pelvis $(89.5 \%)$, followed by the spine, clavicle/scapula/sternum/ribs, skull, and long bones. Of the 38 POEMS syndrome 
46 patients, $35(94.6 \%)$ had osteosclerotic and $32(86.5 \%)$ had mixed lesions. Osteosclerotic lesions

47 were typically scattered, variable in size, and plaque-like, whereas mixed lesions were pouch-

48 shaped or soup bubble-like with a clear sclerotic margin and were generally larger. Although the

49 majority of bone lesions were small in size, $23(62.2 \%)$ had at least one lesion $>10 \mathrm{~mm}$. There

50 was no correlation between serum marker levels and bone lesion patterns after Bonferroni

51 correction (all $\mathrm{P}>0.001)$.

52 Conclusions. $\mathrm{CT}$ is more sensitive and accurate than SS in detecting bone lesions in POEMS

53 syndrome. 
57

Polyneuropathy, organomegaly, endocrinopathy, monoclonal protein, and skin change (POEMS) syndrome is a rare plasma cell disorder. Early diagnosis is challenging since the syndrome is rare and can easily be mistaken for other disorders. Both mandatory major criteria (polyneuropathy and monoclonal plasma cell proliferative disorder), one of the three major criteria [Castleman disease, osterosclerotic lesions, elevated serum levels of plasma vascular endothelial growth factor (VEGF)], and at least one of the six minor criteria are required for a definitive diagnosis. The diagnostic criteria of the Mayo clinic were revised in 2007, when the presence of pathognomonic osteosclerotic lesion was upgraded from minor to major criterion (Li et al., 2013). Bone lesions are important not only for diagnosing and evaluating POEMS syndrome, but are also crucial for administering appropriate treatments (Dispenzier, 2014). Focal radiation is recommended for isolated bone lesions, but systemic chemotherapy should be used in cases of multiple lesions. Therefore, imaging is indispensable for accurately diagnosing POEMS syndrome and determining the treatment approach that is required. However, there is no consensus on the best imaging modality for identifying bone lesions (Minarik et al., 2012). X-ray skeletal survey (SS) and computed tomography (CT) are the two most commonly used methods in clinical practice, but there have been few systematic investigations comparing the effectiveness of the two methods. Most studies are case reports or have examined a small patient sample (Glazebrook et al., 2015; Shi et al., 2015; Shibuya et al., 2011), in part due to the rarity and complexity of this disorder.

Although bone lesions involve diffuse infiltration of light chain-restricted plasma cells 
(Nakajima et al., 2007), POEMS patients present with neither bone pain nor pathologic fractures, which is a major feature of POEMS syndrome that distinguishes it from multiple myeloma. However, the clinical implications of bone lesions and whether they correspond to disease severity or the levels biochemical markers of bone metabolism or inflammatory factors remain open questions.

In this study, we compared the effectiveness of SS and CT for investigating bone lesions in Chinese POEMS syndrome patients. The relationship between bone lesion features from CT images and serum marker levels was also examined.

\section{METHODS}

\section{Patient population}

This was a retrospective study approved by Institutional Review Board, thus the requirement for informed consent was waived. All Chinese patients diagnosed with POEMS syndrome in our medical center from January 2013 to January 2015 were assessed for eligibility. Inclusion criteria were as follows: (a) patients met the 2007 diagnostic criteria for POEMS syndrome; (b) patients underwent an X-ray SS for bone lesions; (c) a chest/abdomen/pelvic CT scan was performed within 1 month of SS; and (d) all SS and CT examinations were completed before treatment. The percentage of plasma cells in bone marrow smears as well as type of immunoglobulin, platelet (Plt), alkaline phosphatase (ALP), serum calcium, phosphate, parathyroid hormone $(\mathrm{PTH})$, beta-isomerized C-telopeptide ( $\beta$-CTx), VEGF, and interleukin (IL)-6 levels were also recorded. The patients' personal information was de-identified prior to 
98 analysis.

\section{Image scanning and interpretation}

The conventional SS included skull, cervical/thoracic/lumbar spine, pelvis, bilateral humeri and

femora. The CT scan range was from the upper margin of sternum to the ischium, and the slice

thickness was 7mm. Two radiologists (FD.W. with 6 years' experience in general diagnostic

radiology and Y.Z. with 15 years' experience in musculoskeletal specialization) evaluated the

bone lesions on a consensus basis using the Centricity PACS system (GE Healthcare, Barrington,

IL, USA). When CT images were evaluated, the bone window was adjusted to a width of 2000

HU and a level of $350 \mathrm{HU}$. The number of bone lesions was recorded by location.

and $>10 \mathrm{~mm}$ ) and appearance (lytic, sclerotic, and mixed). Size was measured based on

maximum lesion diameter. According to previous studies (Glazebrook et al., 2015), an

osteosclerotic lesion had high density surrounded by normal bone marrow; an osteolytic lesion

112 had low density; and a mixed lesion had a central lytic component and was surrounded by a

113 sclerotic margin (Fig. 1).
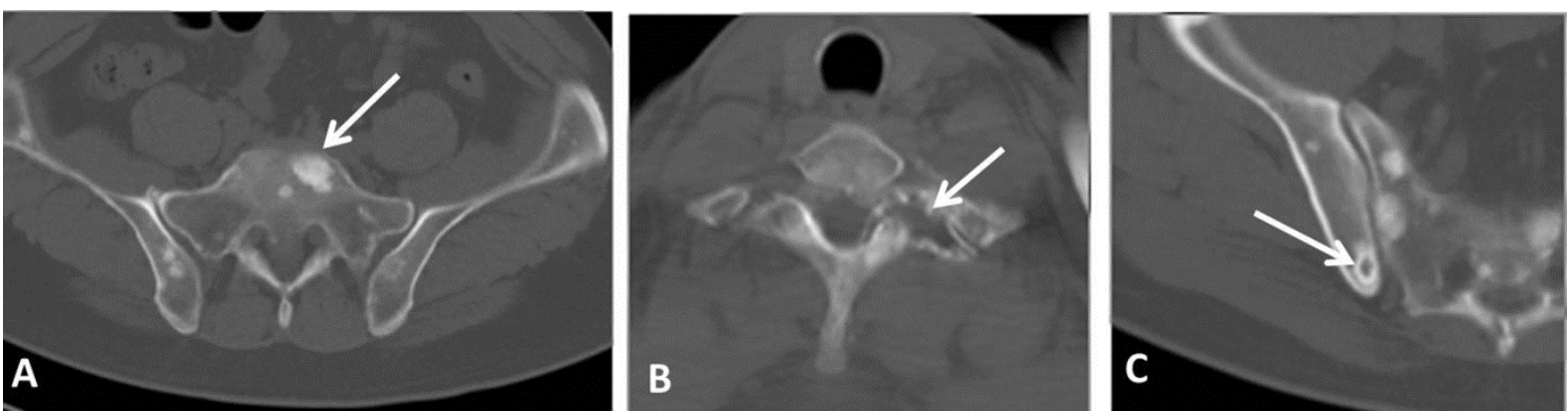

Figure 1 Three types of bone lesions illustrated in CT images. (A) Osteosclerotic lesion; (B) 
116

117

118

119

120

121

122

123

124

125

126

127

128

129

130

131

132

133

134

135

136

Osteolytic lesion; (C) Mixed lesion.

\section{Statistical analysis}

The Paired t test was used to compare the number of bone lesions revealed by SS and CT. A P value $<0.05$ was considered statistically significant. The Pearson correlation was used to evaluate the relationship between the number of bone lesions and serum marker levels.

Bonferroni correction was achieved when P-values were less than $0.001(0.05 / 42)$ in the correlation analysis. Data were analyzed using SPSS v.18.0 software (SPSS Inc., Chicago, IL, USA).

\section{RESULTS}

\section{Study population}

From January 2013 to January 2015, a total of 38 Chinese patients diagnosed with POEMS syndrome according to the 2007 diagnostic criteria were included: 26 males and 12 females, with a mean age of 50.5 years (range: $21-70$ years). The immunoglobulin heavy chain isotypes were $\operatorname{IgA}$ in 25 patients $(65.8 \% ; 25 / 38)$ and $\operatorname{IgG}$ in 13 patients $(34.2 \% ; 13 / 38)$, and the light chain isotypes were $\lambda$ in 35 patients $(92.1 \% ; 35 / 38)$ and $\kappa$ in 3 patients $(7.9 \% ; 3 / 38)$. All the 38 patients underwent bone marrow biopsy, and their Plt, serum calcium, phosphate, ALP, PTH, $\beta$ CTx, and VEGF levels were examined. Serum IL-6 levels were evaluated in 25 patients. The average percentage of plasma cells in bone marrow biopsies was $1.0 \%$ (range: $0 \%-5.5 \%$ ). Levels of other serum markers were as follows: Plt $335 \times 10^{9} / \mathrm{L}$ (range: $89-628 \times 10^{9} / \mathrm{L}$ ); ALP $82.7 \mathrm{U} / \mathrm{L}$ 
137 (range: 50-135 U/L); calcium, $2.13 \mathrm{mmol} / 1$ (range: 1.80-2.40 mmol/l); phosphate, $1.42 \mathrm{mmol} / 1$

138 (range: $1.10-1.90 \mathrm{mmol} / \mathrm{l}$ ); PTH, $30.16 \mathrm{~g} / \mathrm{ml}$ (range: $3.0-101.0 \mathrm{~g} / \mathrm{ml}$ ); $\beta$-CTx, $1.16 \mathrm{ng} / \mathrm{ml}$ (range:

$1390.30-3.50 \mathrm{ng} / \mathrm{ml}$ ); VEGF, 5972 ng/l (range: 534-14328 ng/l); and IL-6, 6.4 pg/ml (range: 2.0-

$14016.0 \mathrm{pg} / \mathrm{ml})$. There were 23 patients with thrombocytosis, while none of the ALP was elevated.

141

142

SS vs. CT

Of the 38 Chinese patients diagnosed with POEMS syndrome, 33 (86.8\%) were found to have

144276 bone lesions by SS. The most commonly affected location was the pelvis $(71.1 \% ; 27 / 38)$,

145 with up to 135 lesions identified in this bone; this was followed by spine $(47.3 \%, 18 / 38)$,

146 cranium $(36.5 \%, 14 / 38)$, and extremities $(28.9 \%, 11 / 38)$. An analysis of chest/abdomen/pelvis

147 CT images revealed 37 patients $(97.4 \%)$ with lesions in these bones. The scope of

148 chest/abdomen/pelvis CT scans included only thoracic/lumbar spine, pelvis, and other flat bones

149 (clavicle/scapula/sternum/ribs). Within this scope, there was no patient whose bone lesion was

detected by X-ray but not by CT. The total number of bone lesions detected by CT was 994 ,

151

which was far greater than the number detected by SS (276 lesions). As detected by SS, the

pelvis was the most commonly affected location in CT scans $(89.5 \% ; 34 / 38)$. The number of

153 patients with lesions in the thoracic or lumbar spine or other bones was $20(52.6 \%), 19(50.0 \%)$,

and 20 (52.6\%), respectively, based on CT scans. The distribution of bone lesions by both

methods is shown in Figure 2. 


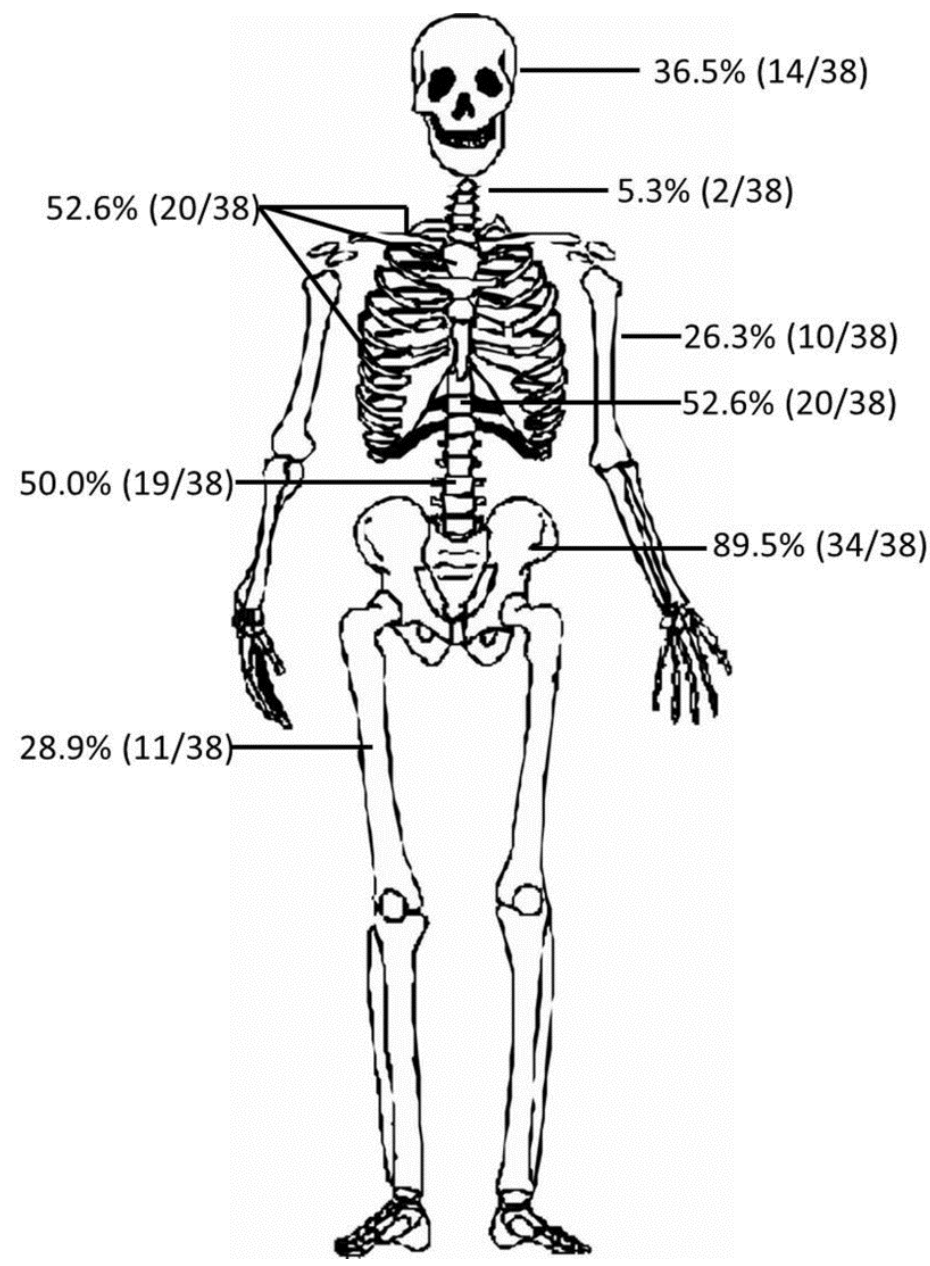

Figure 2 Distribution of bone lesions in POEMS syndrome. The pelvis was the most commonly

affected region, followed by spine, clavicle/scapula/sternum/ribs, cranium, and long bones (humeri and femora). Percentages of patients with affected cranium, cervical spine, and long bones were based on the SS; the others were based on CT images. number of patients with bone lesions detected by each of these imaging methods was compared. At all three locations, more bone lesions were detected by CT than by SS (thoracic spine, $\mathrm{P}=$ 0.001; lumbar spine, $\mathrm{P}=0.003$; pelvis, $\mathrm{P}=0.001$ ) (Fig. 3). In some cases, even after bone lesions 
166 were identified in CT images it was not possible to detect the lesions in SS films obtained from

167 the same patient (Fig. 4). CT was especially useful for detecting small bone lesions and clearly 168 showing their outlines.

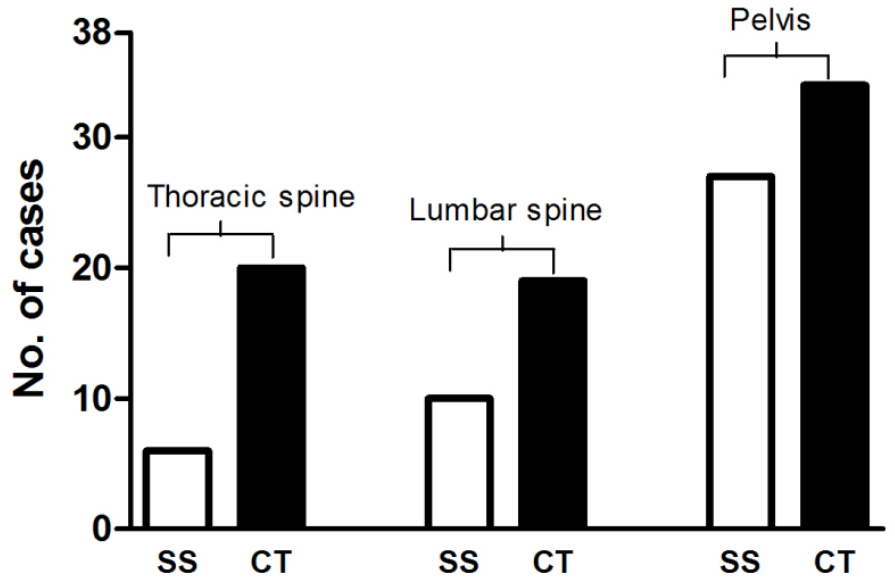

Figure 3 Comparison of the number of patients with bone lesions revealed by SS and CT.
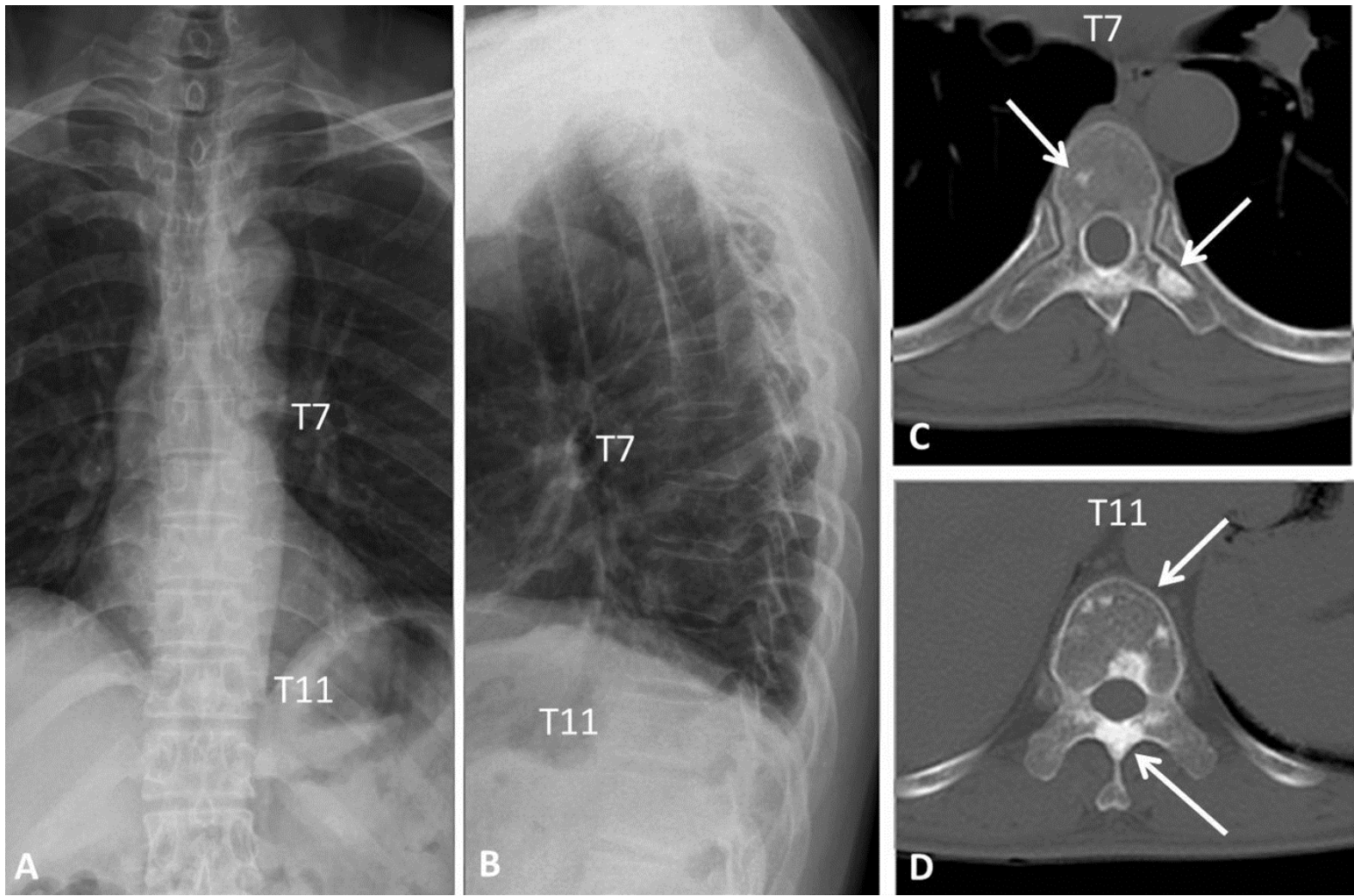

Figure 4 Thoracic spine of a 54-year-old male with POEMS syndrome. Antero-posterior (A) and 
lateral thoracic (B) spine X-rays were interpreted as normal. Sclerotic lesions of the vertebral body and appendix (C, D) were clearly observed by CT (arrows).

175

176

\section{Characteristics of bone lesions in CT images}

The size of each bone lesion in CT images was measured in order to further characterize bone manifestations of POEMS syndrome. Nearly $90 \%$ of patients had bone lesions smaller than 10 mm (<5 mm: 86.5\%, 32/38; 5-10 mm: 89.2\%, 33/38) (Fig. 5A). However, 23 patients (62.2\%)
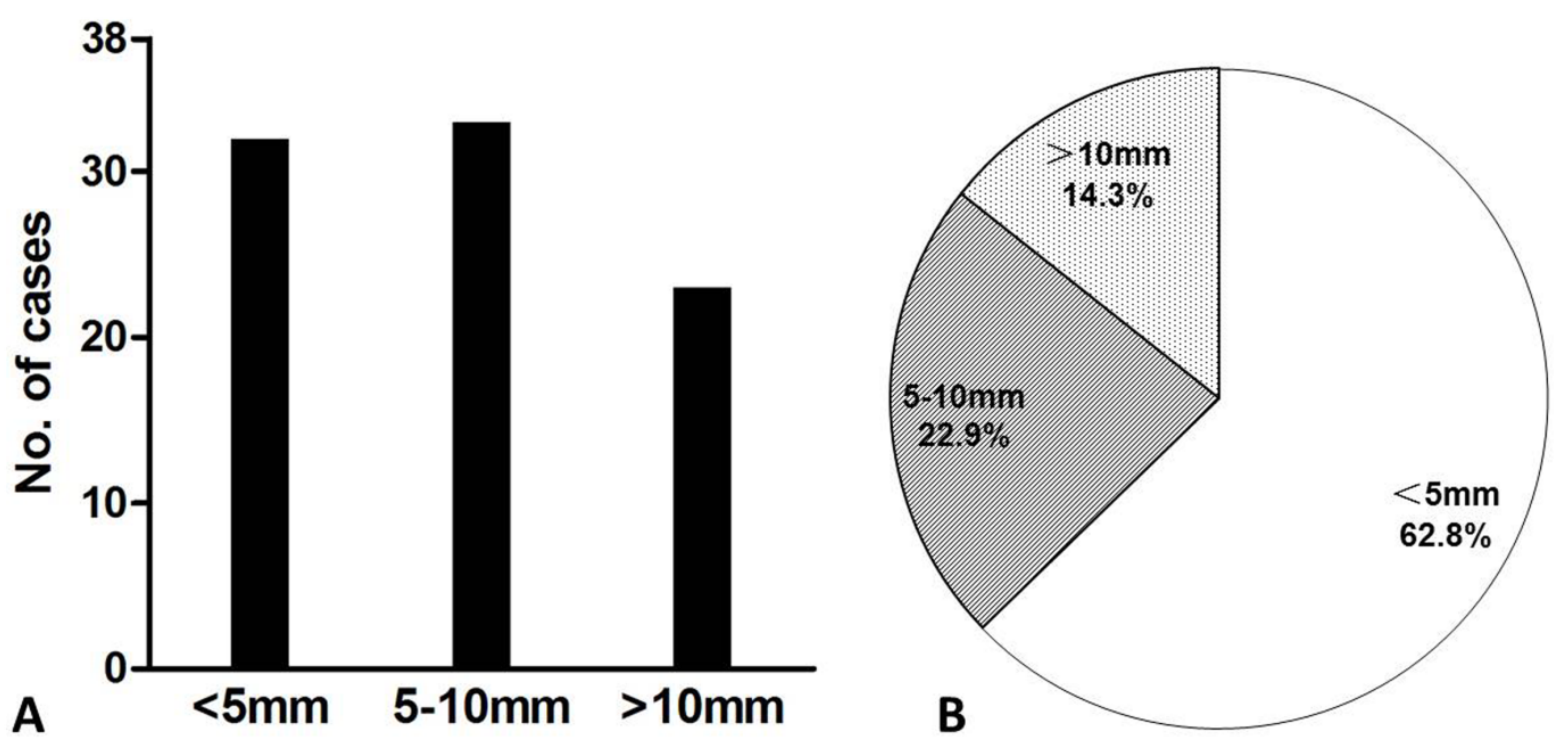

182

Figure 5 Number of patients (A) and bone lesions (B) with respect to lesion size. 


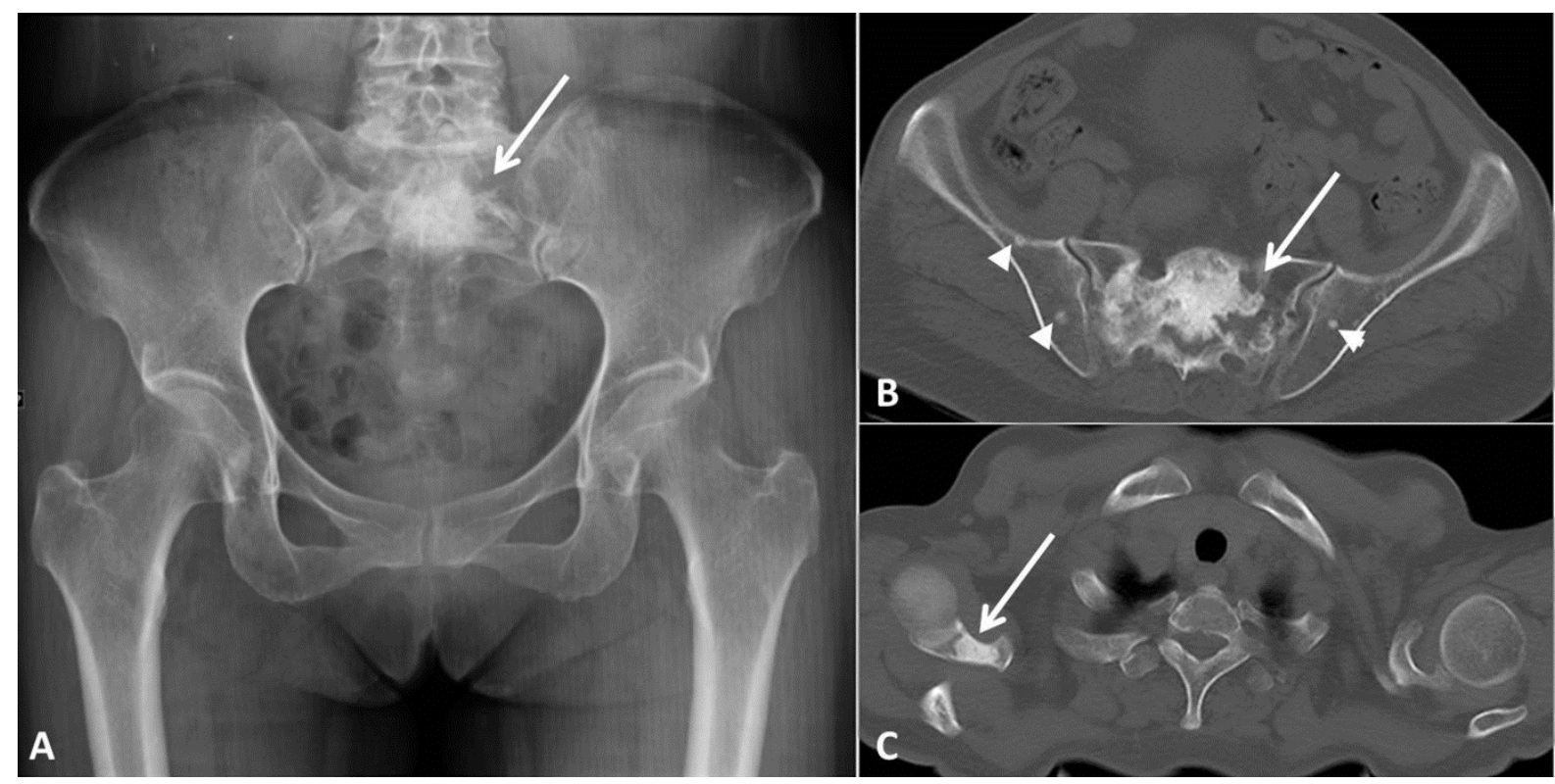

Figure 6 Massive bone lesion of a 56-year-old female with POEMS syndrome. A large (76-mm

diameter) mixed lesion was detected in the sacrum (A and B, arrows). The osteolytic component of the

lesion was clearly visible in the CT image (B). Several small osteosclerotic lesions in the ilium were also more evident in the CT image (B, arrow heads). A sclerotic lesion was detected in the right scapula (C, arrow).

\section{mixed lesions (Fig. 7A). Osteosclerotic lesions were typically scattered, variable in size, and} plaque-like (Fig. 8), whereas mixed lesions were pouch-shaped or soup bubble-like with a clear sclerotic margin and were generally larger (Fig. 9). Patients with pure osteolytic lesions accounted for $15.8 \%(6 / 38)$ of cases. Osteosclerotic lesions were the most prevalent type of bone lesions, accounting for $76.9 \%(764 / 994)$ of the total, followed by mixed $(22.5 \%)$ and osteolytic $(0.6 \%)$ types (Fig. 7B). 

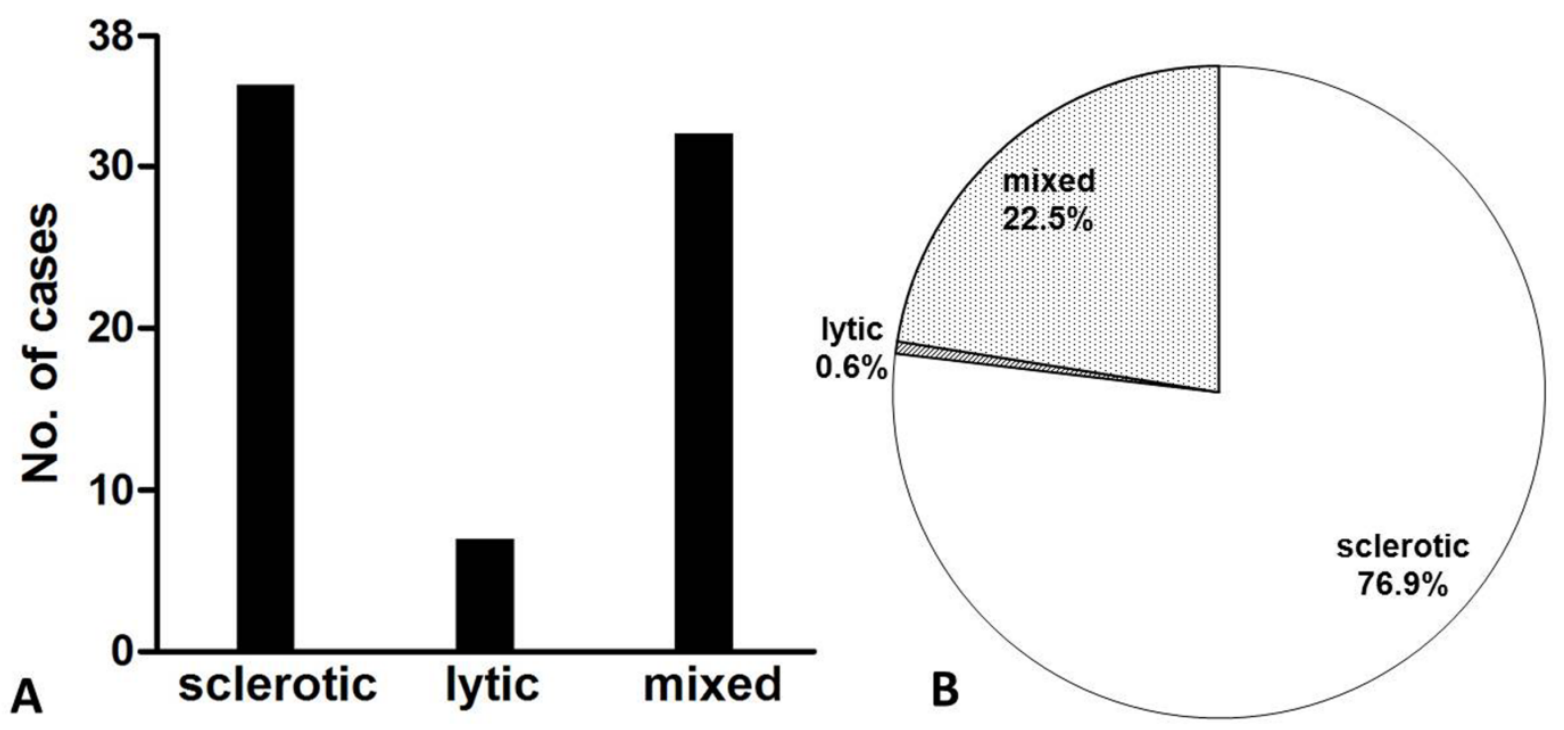

Figure 7 Number of patients (A) and bone lesions (B) as a function of lesion appearance.
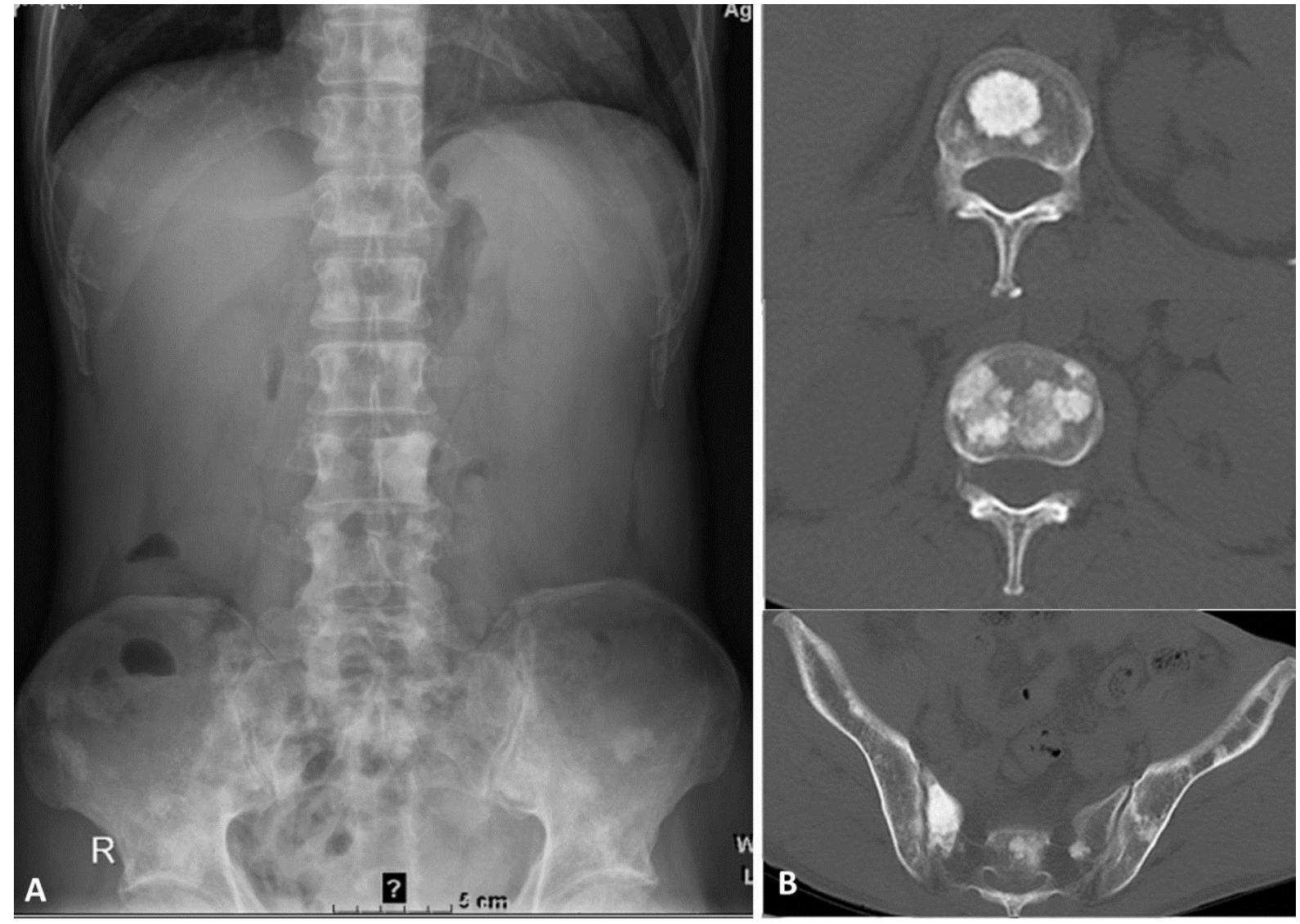

Figure 8 Osteosclerotic lesions in the spine and pelvis of a 53-year-old male with POEMS syndrome.

X-ray (A) and CT (B) images showing multiple plaque-like sclerotic bone lesions in the lumbar spine, 
sacrum, and ilium.
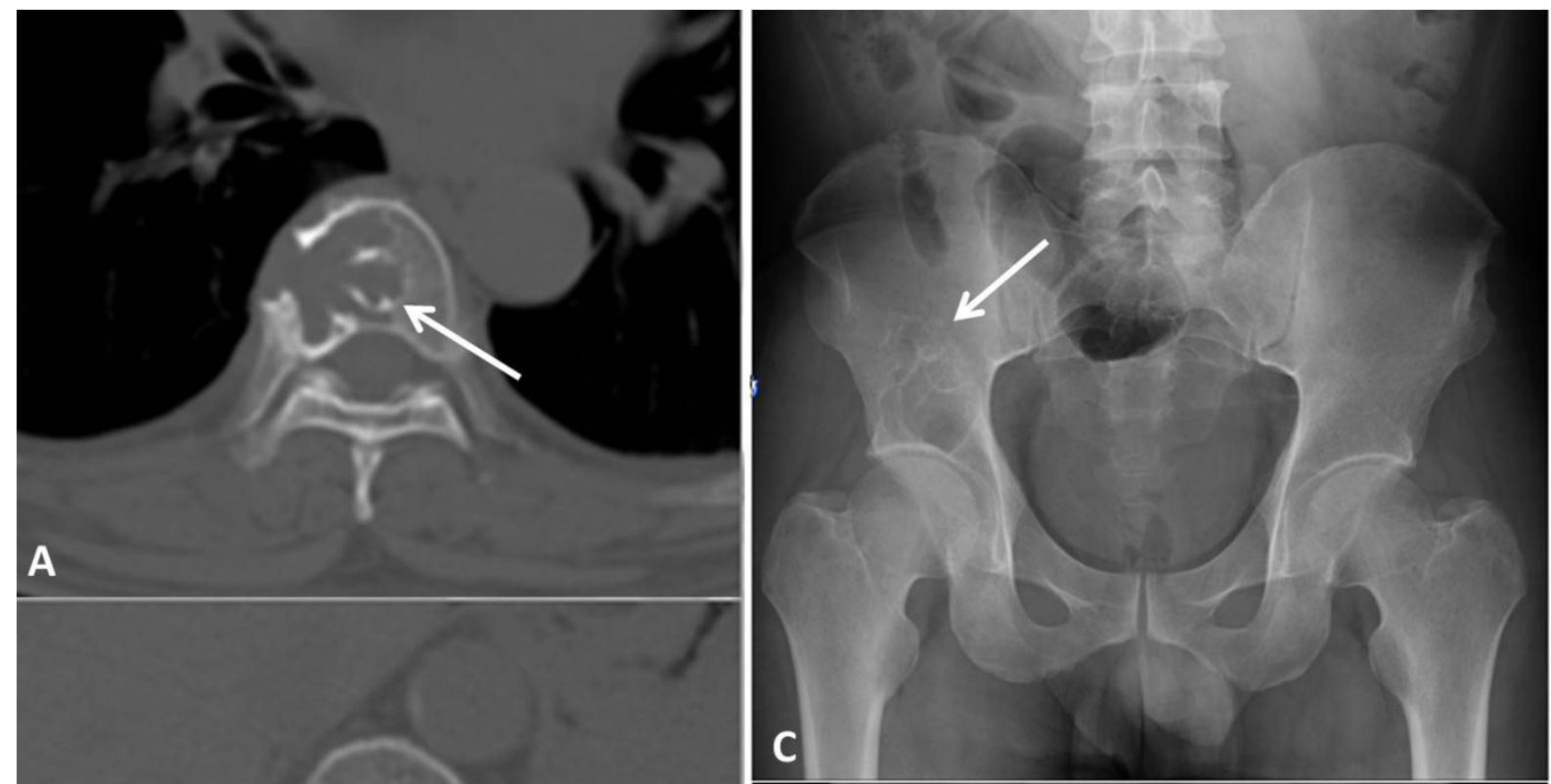

Figure 9 Typical mixed bone lesions of POEMS syndrome. Mixed lesions in the thoracic vertebrae (A,

B) and right ilium (C, D) showing typical pouch and soup-bubble like appearance with a clear sclerotic

rim (arrows). Note the broken bone cortex of the affected vertebral body (A).

Relationship between bone lesions and serum marker levels

210 The relationship between bone lesions and the percentage of plasma cells in bone marrow, as

211 well as serum marker levels (calcium, phosphate, PTH, $\beta$-CTx, VEGF, and IL-6) were detected.

212 However, after Bonferroni correction, none of these markers had a significant correlation with 
213 the number, size, nor type of the bone lesions. (Table 1)

214 Table 1 Correlation between lab markers and bone lesion features in POEMS syndrome.

\begin{tabular}{|c|c|c|c|c|c|c|c|}
\hline \multirow{2}{*}{ Lab markers } & \multirow{2}{*}{ Mean \pm SD } & \multicolumn{6}{|c|}{$P$ value with bone lesion features ${ }^{b}$} \\
\hline & & Total & $<5 \mathrm{~mm}$ & $5-10 \mathrm{~mm}$ & $>10 \mathrm{~mm}$ & Sclerotic lesion & Mixed lesion \\
\hline Plasma cells ${ }^{\mathrm{a}}(\%)$ & $0.96 \pm 1.23$ & 0.785 & 0.987 & 0.509 & 0.568 & 0.873 & 0.692 \\
\hline Calcium (mmol/l) & $2.13 \pm 0.15$ & 0.824 & 0.876 & 0.620 & 0.997 & 0.802 & 0.148 \\
\hline Phosphate (mmol/l) & $1.42 \pm 0.18$ & 0.723 & 0.705 & 0.864 & 0.673 & 0.676 & 0.932 \\
\hline PTH $(\mathrm{g} / \mathrm{ml})$ & $30.16 \pm 20.3$ & 0.752 & 0.592 & 0.906 & 0.974 & 0.959 & 0.393 \\
\hline$\beta-C T x(n g / m l)$ & $1.16 \pm 0.66$ & 0.518 & 0.600 & 0.408 & 0.521 & 0.507 & 0.866 \\
\hline VEGF (ng/ml) & $5972 \pm 3371$ & 0.284 & 0.209 & 0.410 & 0.676 & 0.153 & 0.581 \\
\hline IL-6 (pg/ml) & $6.4 \pm 4.0$ & 0.401 & 0.419 & 0.292 & 0.711 & 0.316 & 0.889 \\
\hline
\end{tabular}

215

a. The percentage of plasma cells in bone marrow biopsies.

b. $\mathrm{P}$ value between lab markers and bone lesion features. After Bonferroni correction, P-values of less than $0.001(0.05 / 42)$ were considered statistically significant. The relationship between lab markers and osteolytic lesions was not analyzed here since there were only 6 patients with this kind of bone lesion.

PTH: parathyroid hormone; $\beta$-CTx: beta-isomerized C-telopeptide; VEGF: vascular endothelial growth factor; IL-6: interleukin 6.

\section{DISCUSSION}

This study retrospectively analyzed bone lesions of 38 Chinese POEMS syndrome patients who underwent SS and chest/abdomen/pelvis CT scans. CT identified more patients and detected more bone lesions than SS (97.4\% vs. 86.8\% and 994 vs. 276 lesions, respectively). Moreover, smaller lesions were detected by CT, which revealed details of the lesions and delineated their margins. Although most (90\%) bone lesions were $<10 \mathrm{~mm}, 62.2 \%$ of patients had at least one bone lesion $>10 \mathrm{~mm}$. Similar numbers of patients had osteosclerotic and mixed lesions $(94.6 \%$ 
229

and $86.5 \%$, respectively). There was no correlation between bone lesion features determined from images and serum levels of metabolic markers or inflammatory factors.

Our finding that, within the thoracic/abdominal/pelvic region, CT is superior to SS for detecting bone lesions is consistent with previous studies. The results suggest that CT may play a dominant role in diagnosis of POEMS, and may replace SS for evaluation of the disease in the future. One study of 24 POEMS patients examined by both CT and SS found a false negative rate of 36\% using the latter method (Glazebrook et al., 2015), while another study arrived at similar conclusions by confirming that $\mathrm{CT}$ was able to detect small bone lesions that were not apparent in the SS (Shi et al., 2015). Some Chinese researchers have reported a much lower rate of affected bones (27\%-55\%) (Cao et al., 2014; Cui et al., 2011; Li et al., 2011; Zhang et al., 2010) than studies from Western countries (86\%-96\%) (Dispenzieri et al., 2013; Dispenzieri et al., 2003). This discrepancy may partially be attributable to the fact that only X-ray scans were used in the Chinese studies.

This finding may have some clinical implications. First, CT may identify bone lesions which SS is not able to show, which constitute a major criterion of POEMS syndrome. Therefore, this will help to diagnose the rare disorder especially for some complicated cases. Second, if SS detects isolated bone lesion, focal radiation is the choice. However, if $\mathrm{CT}$ identifies more bone lesions than SS, the treatment strategy should be changed to systemic chemotherapy (Humeniuk, et al., 2013). Nevertheless, it is unfeasible to conduct whole body CT scans because of concerns of radiation dose; yet it is also unnecessary to obtain a SS if chest/abdomen/pelvis CT is used concurrently. POEMS syndrome patients must undergo a chest/abdomen/pelvis CT in order to 
250

determine whether other features of the disorder such as extravascular overload, organomegaly, and enlarged lymph nodes are also present (Shi et al., 2016). We therefore suggest that a CT bone window is always used for POEMS patients to identify bone lesions, while X-ray SS is used adjunctively to detect lesions specifically in the skull and long bones. Furthermore, as CT could detect more and smaller bone lesions than X-ray, it seems not reasonable to give radiation to a patient with only one or two small $(<10 \mathrm{~mm})$ bone lesions. Therefore, the treatment strategy used nowadays which is mainly based on the number of bone lesions might need to be reconsidered.

Though the majority of bone lesions are osteosclerotic and $<10 \mathrm{~mm}$, mixed and larger lesions require more attention. An early study described prominent osseous proliferation in the spine of three POEMS syndrome patients (Resnick et al. 1981), but this is seldom seen nowadays since patients are diagnosed and treated much earlier than before as a result of advances in diagnostic techniques. Osteosclerotic lesions are pathognomonic for POEMS syndrome and are one of the major diagnostic criteria (Dispenzieri 2014). However, we agree with other reports that mixed lesions with a soap-bubble or pouched appearance are also typical of this disorder (Glazebrook et al., 2015). Therefore, mixed lesions could also be included as diagnostic criteria in the future.

In this study we found that up to $86.5 \%$ of the patients had at least one mixed lesion, which means that Chinese patients share the similar bone lesion features of Western ones. Two previous Chinese studies did not report any mixed lesions with a soap-bubble or pouched appearance (Shi et al., 2015; Shi et al., 2016), possibly because of the small number of patients 
271 (22 and 24 patients), some of whom were included in both studies. Mixed lesions also tend to be

272 larger than other types, with the central lytic component showing increased intensity on T2-

273

274

275

276

277

278

279

280

281

282

283

284

285

286

287

288

289

290

291

weighted sequences of magnetic resonance imaging (MRI) (Nakayama-Ichiyama et al., 2012),

and higher activity in 18F-fluorodeoxyglucose positron emission tomography (PET) (Glazebrook

et al., 2015; Minarik et al., 2012). This may aid in the selection of an appropriate treatment and

monitoring of disease progression.

The relationship between bone lesions and clinical markers of POEMS syndrome is not

well understood. Osteosclerotic bone lesions are characterized by the diffuse infiltration of light

chain-restricted plasma cells (Nakajima et al., 2007; Nakayama-Ichiyama et al., 2012). Bone

marrow aspirates and biopsies from 87 patients with POEMS syndrome demonstrated a

constellation of $\gamma$-restricted monoclonal gammopathy plasma cell rims around lymphoid

aggregates in bone marrow (Dao et al., 2011); however, most bone marrow samples were from

bones without lesions. It has been hypothesized that VEGF, a marker of POEMS syndrome, is

expressed by osteoblasts and causes new bone formation (Dispenzieri et al., 2013). One study

found that $61.0 \%$ of patients with high levels of serum VEGF ( $>2000 \mathrm{ng} / \mathrm{l})$ had osteosclerotic

lesions (Cao et al., 2014), but this study employed only SS and may therefore have

underestimated the number of lesions. Only three patients in our study had serum VEGF levels $<$

$2000 \mathrm{ng} / \mathrm{l}$; one patient exhibited no bone lesions but had a VEGF level of $4965 \mathrm{ng} / \mathrm{l}$. These

results suggest that VEGF level is unrelated to the type and number of bone lesions.

This study had several limitations. Firstly, the number of patients was still relatively

small due to the rarity of POEMS syndrome; however, our patient sample had more homogeneity 
292 than in most previous studies. Secondly, the pathology of bone lesions was not examined. Since

293 different types of bone lesion have distinct pathological features, this requires further

294 investigation. Thirdly, only thoracic-abdominal-pelvic bone lesions were included in the

295 correlation analysis, which may not represent the total bone disease burden. Lastly, other

296 imaging methods such as MRI, skeletal scintigraphy and PET-CT were not included in our

297 analysis, but their effectiveness relative to X-ray SS and CT should also be considered.

\section{CONCLUSION}

300

301

302

303

304

305

306

307

308

309

310

311

312

313

CT was more sensitive and accurate in detecting bone lesions in POEMS syndrome patients than X-ray SS, but the latter method was useful for identifying lesions in the skull and extremities.

Finally, bone lesions in POEMS syndrome were not associated with the levels of metabolic markers or inflammatory factors.

\section{Conflicts of Interest}

None declared.

.

\section{REFERENCES}

Cao X, Wang C, Cai H, Duan M, Zhang W, Li T, Zhou D, Li J. 2014. Diagnostic performance and clinical correlation of serum vascular endothelial growth factor levels in patients with newly diagnosed POEMS syndrome. Zhonghua Xue Ye Xue Za Zhi 35:1065-1068 DOI 10.3760/cma.j.issn.0253-2727.2014.12.004.

Cui RT, Huang XS, Shi Q, Tian CL, Liu JX, Pu CQ. 2011. POEMS (polyneuropathy, organomegaly, 
endocrinopathy, M-protein and skin changes) syndrome in China. Internal Medicine Journal 41:481485 DOI 10.1111/j.1445-5994.2010.02172.x.

Dao LN, Hanson CA, Dispenzieri A, Morice WG, Kurtin PJ, Hoyer JD. 2011. Bone marrow histopathology in POEMS syndrome: a distinctive combination of plasma cell, lymphoid, and myeloid findings in 87 patients. Blood 117:6438-6444 DOI 10.1182/blood-2010-11-316935.

Dispenzieri A. 2014. POEMS syndrome: 2014 update on diagnosis, risk-stratification, and management. American Journal of Hematology 89:214-223 DOI 10.1002/ajh.23644.

Dispenzieri A, Buadi FK. 2013. A review of POEMS syndrome. Oncology (Williston Park) 27:12421250 Available at http://www.cancernetwork.com/oncology-journal/review-poems -syndrome.

Dispenzieri A, Kyle RA, Lacy MQ, Rajkumar SV, Therneau TM, Larson DR, Greipp PR, Witzig TE, Basu R, Suarez GA. 2003. POEMS syndrome: definitions and long-term outcome. Blood 101:2496-2506 DOI 10.1182/blood-2002-07-2299.

Glazebrook K, Guerra BF, Johnson A, Leng S, Dispenzieri A. 2015. Computed tomography assessment of bone lesions in patients with POEMS syndrome. European Radiology 25:497-504 DOI 10.1007/s00330-014-3428-y.

Humeniuk MS, Gertz MA, Lacy MQ, Kyle RA, Witzig TE, Kumar SK, Kapoor P, Lust JA, Hayman SR, Buadi FK, Rajkumar SV, Zeldenrust SR, Russell SJ, Dingli D, Lin Y, Leun N, Dispenzieri A. 2013. Outcomes of patients with POEMS syndrome treated initially with radiation. Blood 122:68-73 DOI 10.1182/blood-2013-03-487025.

Li J, Zhou DB. 2013. New advances in the diagnosis and treatment of POEMS syndrome. British 
Journal of Haematology 161:303-315 DOI 10.1111/bjh.12236.

Li J, Zhou DB, Huang Z, Jiao L, Duan MH, Zhang W, Zhao YQ, Shen T. 2011. Clinical characteristics and long-term outcome of patients with POEMS syndrome in China. Annals of Hematology 90:819-826 DOI 10.1007/s00277-010-1149-0.

Minarik J, Scudla V, Bacovsky J, Pika T, Ctvrtlik F, Metelkova I, Myslivecek M. 2012. Comparison of imaging methods in POEMS syndrome. Biomedical Papers of the Medical Faculty of the University Palacky 156:52-57 DOI 10.5507/bp.2011.053.

Nakajima H, Ishida S, Furutama D, Sugino M, Kimura F, Yokote T, Baba I, Tsuji M, Hanafusa T. 2007. Expression of vascular endothelial growth factor by plasma cells in the sclerotic bone lesion of a patient with POEMS syndrome. Journal of Neurology 254:531-533 DOI 10.1007/ s00415-006-0268-y.

Nakayama-Ichiyama S, Yokote T, Hirata Y, Iwaki K, Akioka T, Miyoshi T, Nishiwaki U, Masuda Y, Hiraoka N, Takayama A. 2012. Multiple cytokine-producing plasmablastic solitary plasmacytoma of bone with polyneuropathy, organomegaly, endocrinology, monoclonal protein, and skin changes syndrome. Journal of Clinical Oncology 30:e91-e94 DOI 10.1200/JCO.2011. 38.9288.

Resnick D, Greenway GD, Bardwick PA, Zvaifler N J, Gill G N, Newman DR. 1981. Plasma-cell dyscrasia with polyneuropathy, organomegaly, endocrinopathy, M-protein, and skin changes: the POEMS syndrome. Radiology 140:17-22 DOI 10.1148/radiology.140.1.7244223.

Shi X, Hu S, Luo X, Luo M, You H, Zhu Y, Xi X. 2016. CT characteristics in 24 patients with POEMS syndrome. Acta Radiologica 57:51-57 DOI 10.1177/0284185114564614. 
356 Shi XF, Hu SD, Li JM, Luo XF, Long ZB, Zhu Y, Xi XD. 2015. Multimodal imaging and clinical characteristics of bone lesions in POEMS syndrome. International Journal of Clinical and Experimental Medicine 8:7467-7476 Available at www.ijcem.com/ISSN:1940-5901/IJCEM 0007180.

360

Shibuya K, Misawa S, Horikoshi T, Kanai K, Isose S, Nasu S, Sekiguchi Y, Noto Y, Fujimaki Y, Nakaseko C. 2011. Detection of bone lesions by CT in POEMS syndrome. Internal Medicine 50:1393-1396 DOI 10.2169/internalmedicine.50.5263.

Zhang B, Song X, Liang B, Hou Q, Pu S, Ying JR, Gao C. 2010. The clinical study of POEMS syndrome in China. NeuroEndocrinology Letters 31:229-237 Available at http://www.ncbi.nlm. nih.gov/pubmed/20424579. 